\title{
Orthognathic Surgery Combined with Partial Lipectomy of the Buccal Fat Pad: Case Report on Optimization of Esthetic Outcome
}

\author{
Daniel Amaral Alves Marlière ${ }^{1}$, Caio Bellini Lovisi ${ }^{1}$, Alan Robert Moreira Schmitt ${ }^{2}$, Bruno Salles Sotto-Maior ${ }^{3}$ \\ and Henrique Duque de Miranda Chaves Netto ${ }^{3}$ \\ 1. MSc in Clinical Dentistry - Juiz de Fora Dental School, Federal University of Juiz de Fora, Juiz de Fora 36036-300, Brazil \\ 2. MSc in Dentistry - Dental School, University of the State of Rio de Janeiro, Rio de Janeiro 20551-030, Brazil \\ 3. Lecturer in Dentistry - Juiz de Fora Dental School, Federal University of Juiz de Fora, Juiz de Fora 36036-300, Brazil
}

\begin{abstract}
Orthognathic surgery involves correction of functional and often esthetic disharmonies. The purpose of this case report is to present the case of a 22 years-old female with class III dentofacial deformity that required orthognathic surgery to treat her malocclusion and buccal fat pad excision for optimizing soft-tissue facial contour. Treatment planning involved a counterclockwise rotation of the maxilomandibular complex with genioplasty based on digital 3D surgical planning using the Dolphin Imaging ${ }^{\circledR} 11.7$ 3D Premium software. Prototyping splints allowed surgical procedures (bilateral sagital mandibular osteotomy associated to a Le fort I osteotomy) that corrected position of the gnathic bones. In the same surgical procedure, the buccal fat pad was partially removed for a sharp cheek contour giving a slimmer appearance to her face. After 5 months, the patient was reassessed and reported no complaints regarding esthetics or function. This case illustrates that a combination of orthognathic surgery and an esthetic procedure can result in a stable and harmonious facial as well as occlusal outcomes.
\end{abstract}

Key words: Orthognathic surgery, occlusal plane, malocclusion, buccal fat pad, aesthetic procedure.

\section{Introduction}

Orthognathic surgery aims to correct skeletal and dental discrepancies, providing varying degrees of changes to facial soft tissues. Currently, surgical planning should contemplate symmetry, proportion, and, above all, facial harmony [1]. In order to maximize the benefits of surgery, however, it may be necessary to focus on supporting procedures to optimize esthetics [2].

In order to optimize facial appearance, orthognathic surgery planning should consider which co-operative procedures might be indicated. Among the treatments for facial esthetics optimization, the following may be highlighted: rhinoplasty, V-Y suture for increased vermilion exposure of the upper lip, and paranasal

Corresponding author: Daniel Marlière, DDS, MSc, research fields: orthognathic surgery. filling with alloplastic materials [2].

Since 1977 when Egyedi [3] first recommended the use of the pedicled buccal fat pad to repair oral defects, this flap also has been used for facial aesthetic surgery $[4,5]$. Rubio-Bueno et al. [6] demonstrated a method of manipulating pedicles of the buccal fat pad simultaneously with a Le Fort I osteotomy for maxilla advancement aiming at obtaining paranasal filling and upper labial support in patients with Class III malocclusion, thus highlighting the usefulness of such combined approach for esthetic refinement. Hernández-Alfaro et al. [7] performed a subjective and objective three-dimensional evaluation of six patients with dentofacial anomalies and bilateral malar hypoplasia were managed with simultaneous orthognathic surgery and pedicled buccal fat pad augmentation. In conclusion, the pedicled buccal fat pad technique is a reasonable alternative for malar 
augmentation, and the results suggested that it provides satisfactory soft tissue augmentation, avoided the use of foreign materials, had minimal morbidity, high patient satisfaction, and adequate stability at 12 month follow-up.

Surgical procedures are available to create an apparent refinement of the facial contour and projection of the zygomatic region [8-10]. In this context, it is possible to partially remove the adipose tissue in the superficial portion of the buccal pad in order to reduce the volume of soft tissue below the zygomatic region (prominent cheek).

In this case report, a dentofacial class III deformity correction approach is presented with the association of orthognathic surgery and removal of the fat pad to optimize esthetics in terms of facial contour and refinement.

\section{Case Report}

A healthy 22-year-old female presented to the Oral and Maxillofacial Surgery Clinic of the Federal University of Juiz de Fora for treatment of dentofacial deformity, which was accompanied by complaints of esthetic maxillary deficiency and functional masticatory restrictions. The patient signed an informed consent form for both treatment and use of images for publication [11].

Frontal facial examination showed a long face appearance with no apparent vertical maxillary excess. On smile evaluation, adequate gingival exposure was observed in the anterior region of the maxilla (approximately $2 \mathrm{~mm}$ from the gingival margin). At rest and spontaneous smiling, bilateral prominent cheek was observed in the middle third contour of her face. Regarding her facial profile, a middle third deficiency in paranasal filling and an acute nasolabial angle were observed, together with a lower projection of the chin in relation to the lower lip (Fig. 1A-I). The patient also presented a class III malocclusion $(0 \mathrm{~mm}$ overbite, $-9 \mathrm{~mm}$ horizontal trespass, $2 \mathrm{~mm}$ deviation to the right of the upper midline between central incisors). She reported has been undergoing orthodontic treatment for 2 years.

\subsection{Virtual Planning}

Cone-beam computed tomography was performed

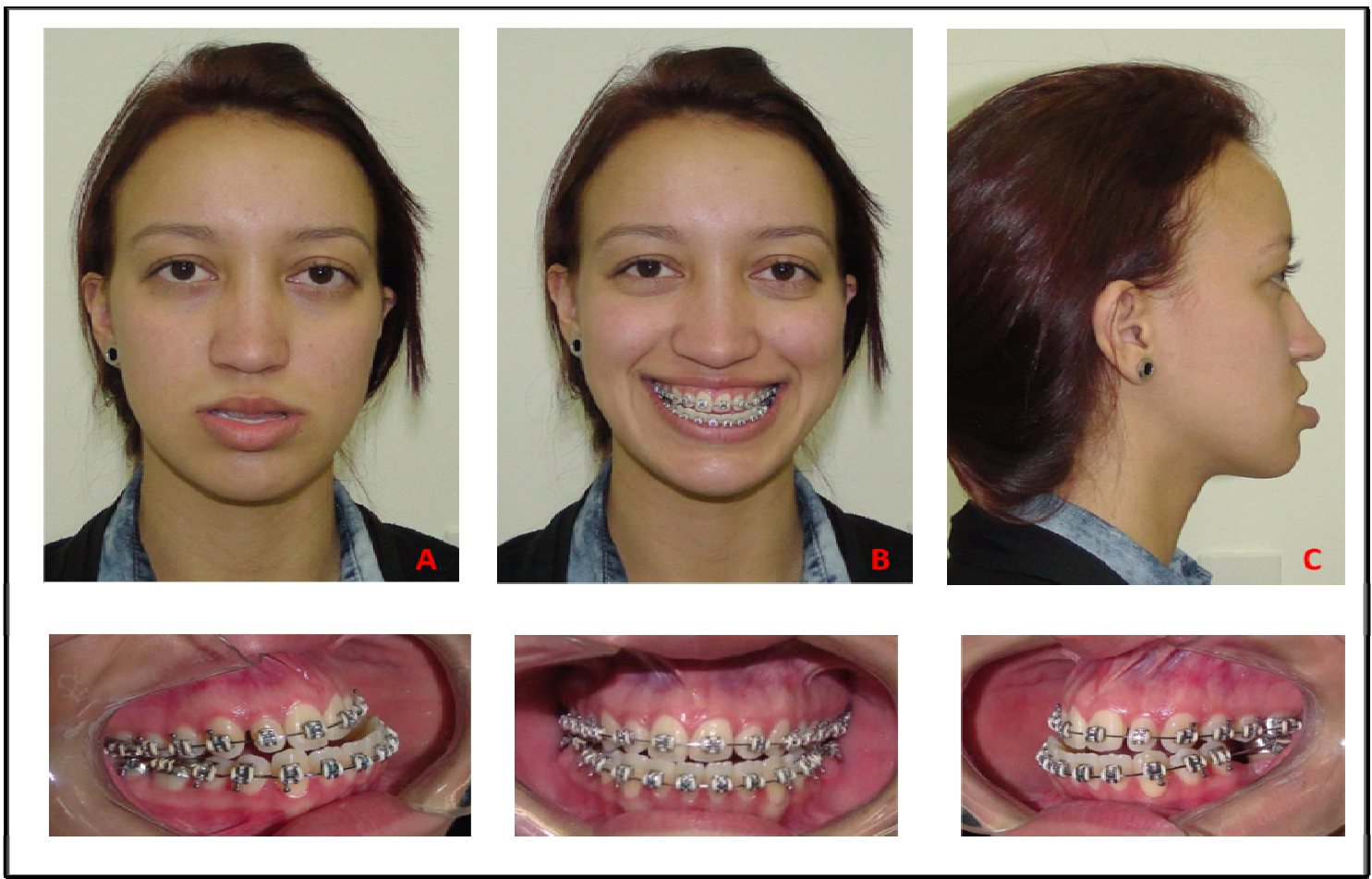

Fig. 1 A-Frontal view; B-Frontal view smiling; C-Lateral view (facial profile). 
using the i-CAT system (Image Sciences International, Hatfield, PA, USA). Three-dimensional images were reconstructed using the Dolphin Imaging ${ }^{\circledR} 11.7$ 3D program (Dolphin Imaging and Management Solutions, Chatworth, CA, USA). The surgical planning was based on the patient's esthetic-functional complaints as well as three-dimensional facial and cephalometric analysis. According to these factors, a bimaxillary (maxilla and mandible) surgical approach was planned based on counterclockwise rotation of the maxillomandibular complex with mentoplasty. The Le Fort I osteotomy was selected for advancement and discreet maxillary impaction in the region of the anterior nasal spine and upper incisors, midline correction to the left in relation to the philtrum, occlusal cant leveling in canines and molars. Sagittal mandibular osteotomy bilaterally was selected for mandibular retreatment and chinplasty for chin advancement (Fig. 2 A-G).

\subsection{Surgical Procedure}

The surgical procedure was performed under general anesthesia. Initially, buccal access to the mandible was achieved through soft tissue incision on the external oblique line to the mesial aspect of the second molar laterally (a minimum of $5 \mathrm{~mm}$ of non-keratinized mucosa maintained in the buccal region). Subperiosteal dissection of the buccal mucosa was then performed towards the internal oblique line in the retromolar region, aiming at partially exposing the medial region and lingula of the mandible. Using reciprocating saws (Stryker-CORE System), sagittal osteotomy of the bilateral mandible was performed and finished using chisels and hammer. Stereolithographic surgical guides were then fixed to the orthodontic appliance for maxilomandibular

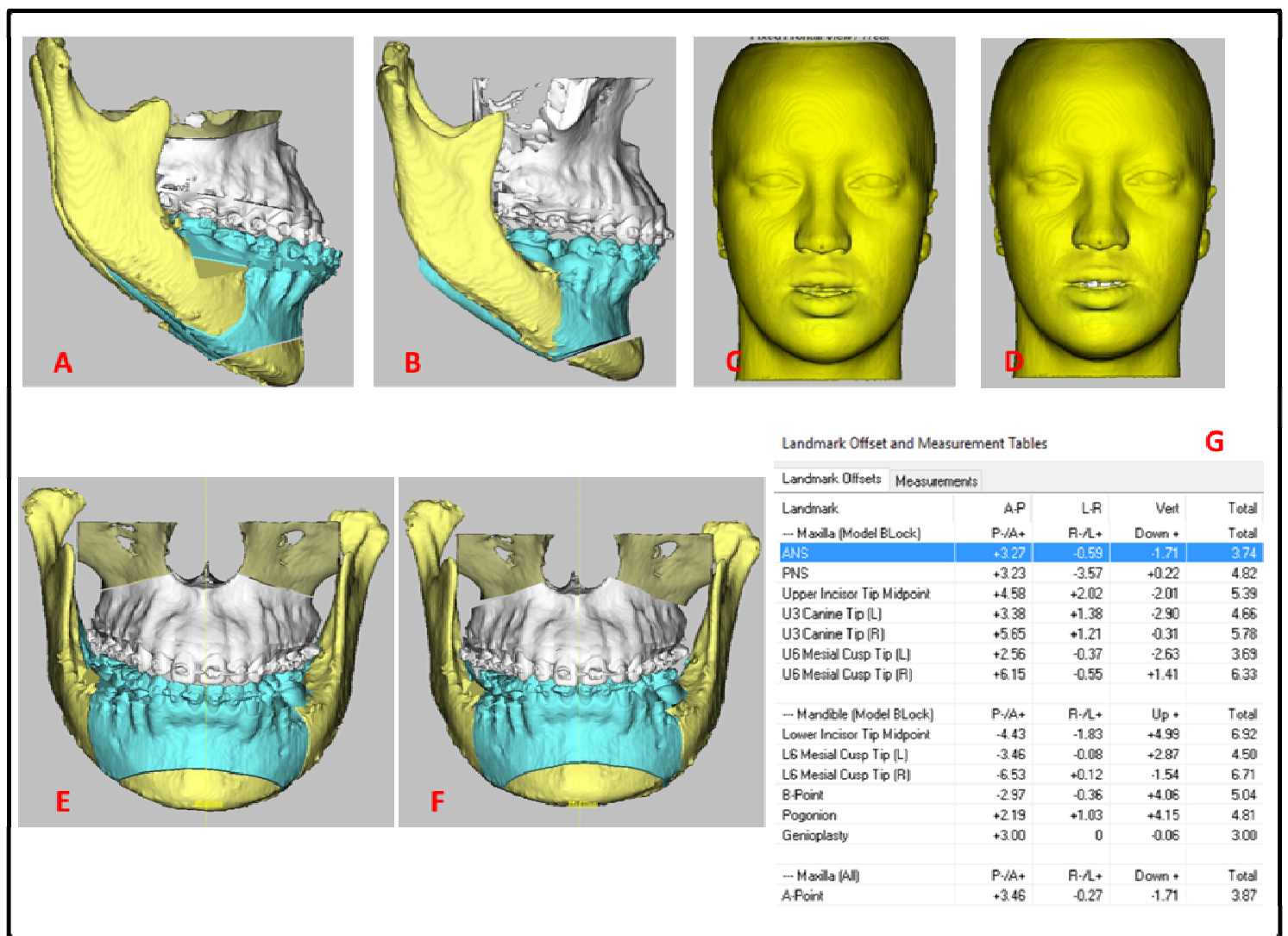

Fig. 2 A and E-3D reconstruction of the maxilla and the mandible prior to simulation of virtual planning; $B$ and $F-3 D$ reconstruction of the maxilla and the mandible after simulation of virtual planning; $\mathrm{C}$ - Soft tissue contour prior to simulation; D-Prediction of facial soft tissue; G-Values in $\mathbf{~ m m}$ of the skeletal and dental movements in the maxilla and mandible. 
splinting with steel wire. The mandible and maxilla were stabilized in intermediate occlusion, and the mandible was repositioned via rigid internal fixation with straight miniplates and monocortical screws (System 2.0—Neoface-Neoortho Orthopedic Products).

Surgical access to the maxilla was performed buccally and subperiosteal non-keratinized mucosa detachment, extending from the floor of the nasal fossa to the pterygomaxillary region. Le Fort I osteotomy was performed using a reciprocating saw (Stryker-CORE System) and finished with chisels and hammer. After osteotomy in the pterygomaxillary regions and mobilization of the maxilla, the walls of the maxilla were leveled following the virtual plan, using rongeur forceps and rotatory burs. The final stereolithographic surgical guide was inserted along with the orthodontic appliances and steel wire to stabilize the maxilla and the mandible in final occlusion. Finally, the maxilla was repositioned with rigid internal fixation using $\mathrm{L}$-shaped miniplates in the zigomaticomaxillary regions and around the pyriform aperture (System 2.0-Neoface-Neoortho Orthopedic Products). Mentoplasty was performed for chin advancement to improve the contour of the mentolabial groove. The surgical procedures followed the virtual treatment planning.

In conjunction with the bimaxillary orthognathic surgery and mentoplasty procedures, partial removal of the buccal adipose pads was performed. After restoration and stabilization of the bone structures, the buccal access flap to the mandible was used to reach the buccal extension of the adipose pad tissue. The buccinator muscle fibers were dissected without incision, only using a curved hemostatic forceps to expose the buccal fat pad. The adjacent fascia was delicately and laterally dissected superficially to facilitate prolapse of part of the adipose pad towards the oral cavity. Due to its proximity to the facial nerve and parotid duct, the fat capsule should be handled with care. By gently pulling the capsule, fat prolapse increased (Fig. 3 A-B). Using this method, the fat pad was easily removed. This lipectomy (excision or partial removal) was considered a coadjuvant procedure, aiming to optimize esthetic outcome through refinement of the facial middle third contour.

The patient was evaluated weekly for the first 2 months and monthly thereafter until the sixth month. Postoperative orthodontic treatment was maintained until completion (Fig. 4 A-I). Subjectively, the patient was satisfied with optimal esthetic and functional result.

\section{Discussion}

In order to correct this class III malocclusion, bimaxillary orthognathic surgery was performed based on the inverted sequence (mandible osteotomy first)

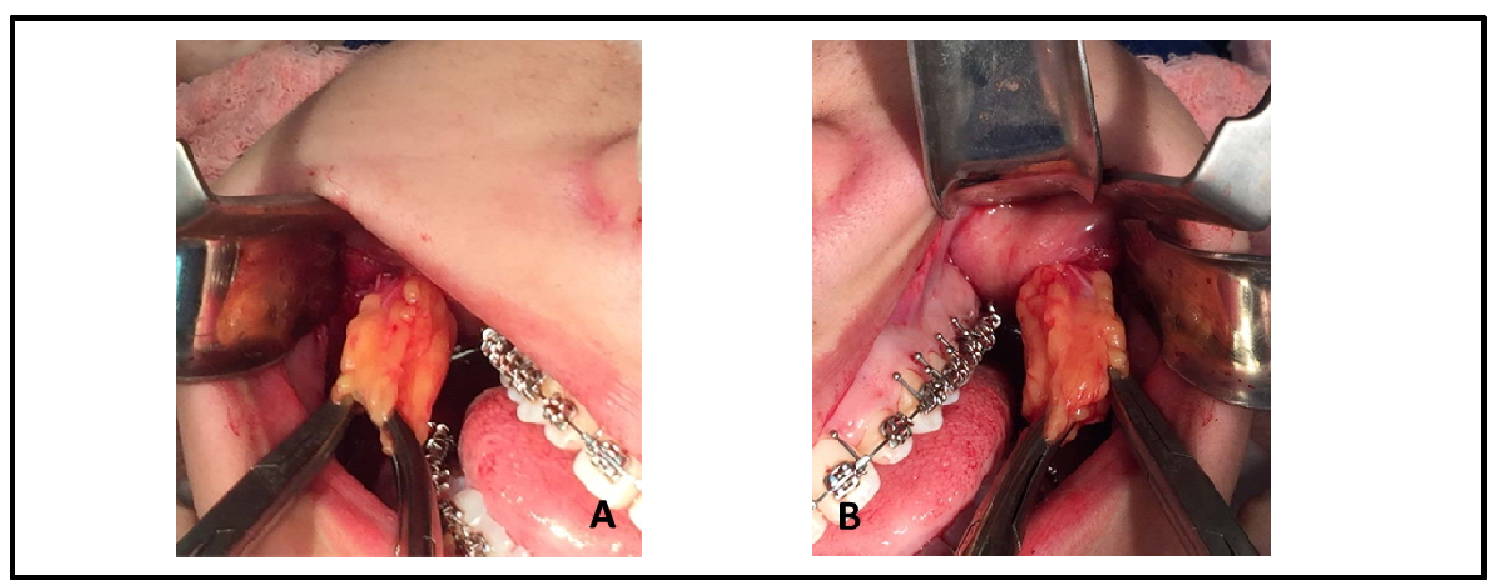

Fig. 3 A-B: After buccal adipose cushion prolapse, traction of fatty tissue for partial removal. 


\section{Case Report on Optimization of Esthetic Outcome}

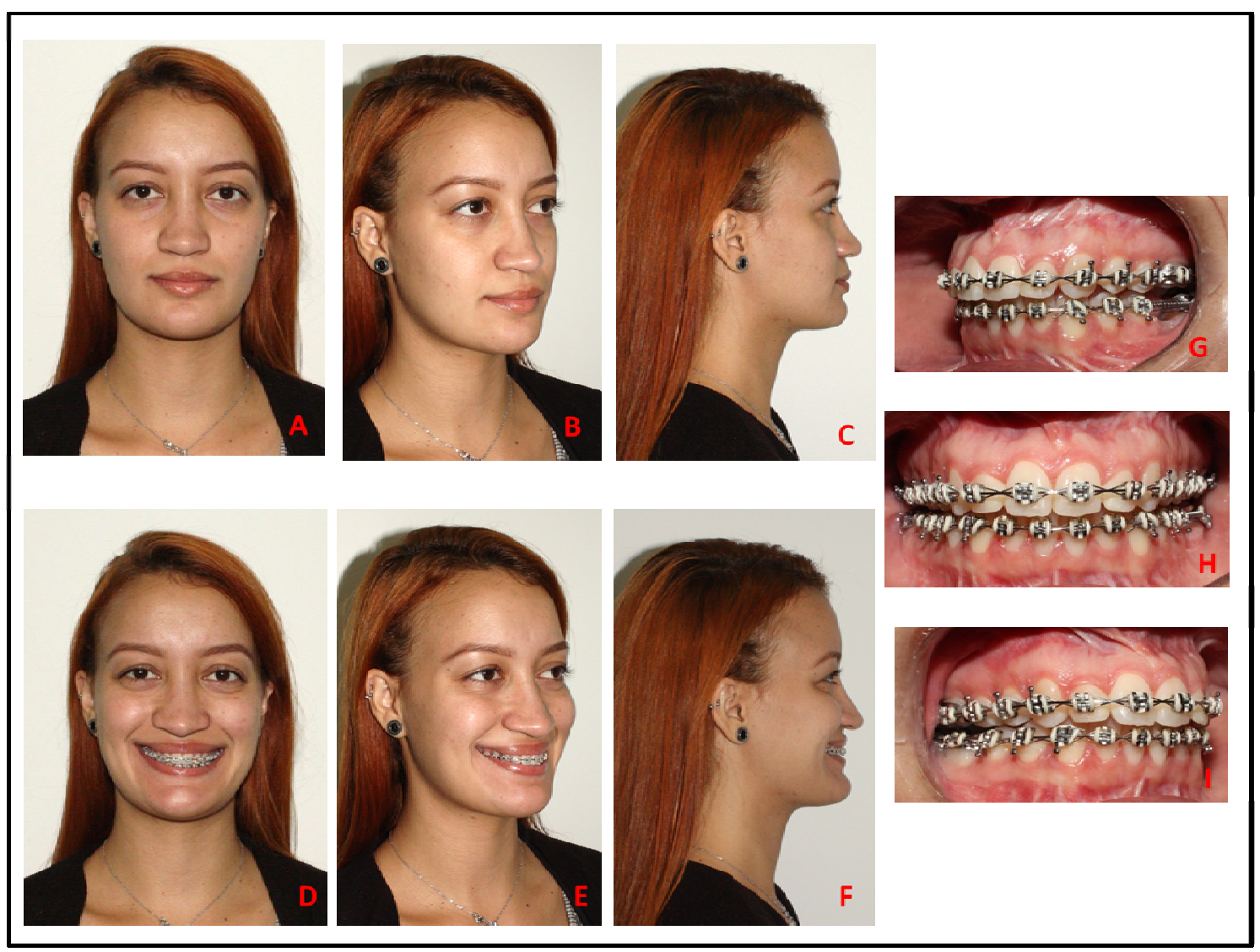

Fig. 4 A-F: Post-operative evaluation (08 months) at rest and smiling. G-I: Intraoral images, postoperative occlusion after orthodontic treatment.

from the rotation of the occlusal plane (maxillomandibular complex) in an counterclockwise direction associated with partial lipectomy of the buccal fat pad.

The traditional sequence of orthognathic surgery is initiated in the maxilla as a preference among surgeons [12]. After diagnosis, facial analysis and planning, osteotomy of both the maxilla and the mandible was deemed necessary, starting from sagittal osteotomy of the mandible bilaterally (inverted sequence). According to Borba et al. [13], orthognathic surgery in the inverted-sequence approach was described in the 1970s, however, to date, the decision regarding such inverted approach is based on the experience and preference of the surgeon. In addition, the inverted surgical sequence would be beneficial in situations such as rotation of the occlusal plane counterclockwise to avoid an anterior open bite intraoperatively (when intermaxillary fixation is impaired by a thick intermediate guide), inaccuracy of intercondylar registration and uncertainty regarding condylar positioning [14].As part of the planning, Posnick et al. [15] highlighted that facial esthetics is enhanced by the clockwise or counterclockwise rotations of the maxillary and/or mandibular planes, as opposed to angular measurements between occlusal plane and skeletal structures postoperatively. The occlusal plane angles may show wide variability within the general population and, therefore, there is no clear functional advantage in obtaining a specific “degree” of angulation.

In this context, Parente et al. [16] also showed that planning in orthognathic surgery should not be based solely on numeric cephalometric values. It should be otherwise based on the surgeon's perceptions and patient's clinical characteristics, namely facial type, upper incisors at rest, teeth exposure when smiling, lip, nasal and paranasal support, projection of the chin in 
relation to the lower lip and lower incisors, and such parameters should be catered for each patient individually.

Therefore, the balance among the 5 aforementioned clinical characteristics described by Parente et al. [16] was used in the case presented herein to achieve a reasonable esthetic outcome. The patient benefited from counterclockwise rotation because the chin rotated anteriorly, the angle of the mandibular plane decreased, the prominence of the mandibular angles increased and the mandibular contour was thereby improved. Based on this concept, it is believed that the counterclockwise rotation moved the mandibular incisors backwards while the chin moved forward, probably reducing the actual mandibular retreat and thus preserving the pharyngeal airway space (Fig. 2G).

Esthetic optimization was achieved using partial lipectomy of the buccal fat pad (partial removal) through the same access window used for the mandibular sagittal osteotomy. In general, such procedure has been reported as an incision $2 \mathrm{~cm}$ above or below the parotid duct in the buccal mucosa at the level of the second upper molar [8-10]. For Rubio-Bueno et al. [6], the buccal fat pad is frequently found accidentally during orthognathic surgery. Therefore, a new incision was not needed for access to the adipose pad and, in addition, greater protection was given to the parotid duct, since dissection was performed safely below it.

Partial removal of the buccal fat pad reduces the prominence of the cheeks and accentuates the zygomatic region, contributing to the zygomatic bone contour in the middle third of the face in patients whose "chubby cheeks" may conceal this feature [8]. According to Matarasso [9], it provides a sculpted facial appearance and refines the mandibular contours.

In order to obtain such an effect, Matarasso [9] speculatively suggested the removal of up to 3 grams of adipose tissue. Hasse and Lemperle [8] suggested the loss of 4 to 5 grams, though no scientific evidence has been found to support either quantity. It is also worth noting that partial removal of the buccal fat pad in the immediate postoperative period may be impaired by transoperative edema [2]. In spite of that, evaluating the facial contour after orthognathic surgery, even with possible unpredictability due to slight immediate facial edema, was deemed acceptable based on clinical judgment and common sense that the adjuvant procedure enhanced facial contour refinement (Fig. 4C-D).

\section{Conclusions}

This case report showed that a stable and harmonic result between facial esthetics and occlusion is possible to achieve combining orthognathic surgery with partial lipectomy of the buccal fat pad.

Subjectively, simultaneous surgical procedures were associated with minimal morbidity and high patient satisfaction. It is clear, however, that scientific evidence is lacking to minimize possible imprecisions of such adjuvant esthetic approach and to ameliorate predictability.

\section{References}

[1] O’Ryan, F., and Lassetter, J. 2011. “Optimizing Facial Esthetics in the Orthognathic Surgery Patient.” J Oral Maxillofac Surg. 69: 702-15.

[2] Mohamed, W. V., and Perenack, J. D. 2014. "Aesthetic Adjuncts with Orthognathic Surgery.” Oral Maxillofacial Surg Clin N Am. 26: 573-85.

[3] Egyedi, P. 1977. "Utilization of the Buccal Fat Pad for Closure of Oroantral and/or Oro-Nasal Commucations.” $J$ Maxillofac Surg. 5: 241-4.

[4] Chung, S. C., Ann, H. Y., Um, I. W., and Kim, C. S. 1999. "Buccal Fat Pad Transfer as a Pedicled Flap for Facial Augmentation.” J Korean Assoc Maxillofac Plast Reconstr Surg. 13: 153-9.

[5] Ramirez, O. M. 1999. "Buccal Fat Pad Pedicle Flap for Midface Augmentation.” Ann Plast Surg. 43: 109-18.

[6] Rubio-Bueno, P., Ardanza, B., Piñas, L., and Murillo, N. 2013. "Pedicled Buccal Fat Pad Flap for Upper Lip Augmentation in Orthognathic Surgery Patients.” J Oral Maxillofac Surg 71: 178-84.

[7] Hernández-Alfaro, F., Valls-Ontañon, A., and Blasco-Palacio, J. C. 2015. "Malar Augmentation with Pedicled Buccal Fat in Orthognatic Surgery: 


\section{Case Report on Optimization of Esthetic Outcome}

Three-dimensional Evaluation.” Plast Reconstr Surg. 136 (5): 1063-7.

[8] Hasse, F. M., and Lemperle, G. 1994. "Resection and Augmentation of Bichat's Fat Pad in Facial Contouring." Eur J Plast Surg. 17: 239-42.

[9] Matarasso, A. 2006. "Managing the Buccal Fat Pad." Aesthetic Surg J. 26: 330-6.

[10] Xu, J., and Yu, Y. J. 2013. “A Modified Surgical Method of Lower-face Recontouring.” Aesth Plast Surg 37: 216-21.

[11] Gagnier, J. J., Kienle, G., Altman, D. G., Moher, D., Sox, H., and Riley, D. 2014. "The CARE Guidelines: Consensus-based Clinical Case Report Guideline Development.” Journal of Clinical Epidemiology 67: 46-51.

[12] Cottrell, D. A., and Wolford, L. M. 1994. "Altered Orthognathic Surgical Sequencing and a Modified Approach to Model Surgery.” J Oral Maxillofac Surg. 52:
1010-20.

[13] Borba, A. M., Borges, A. H., Cé, P. S., Venturi, B. A., Naclério-Homem, M. G., and Miloro, M. 2016. "Mandible-first Sequence in Bimaxillary Orthognathic Surgery: A Systematic Review.” Int J Oral Maxillofac Surg 45: 472-5.

[14] Perez, D., and Ellis, E. 2011. "Sequencing Bimaxillary Surgery: Mandible First.” J Oral Maxillofac Surg 69: 2217-24.

[15] Posnick, J., Fantuzzo, J. J., and Orchin, J. D. 2006. "Deliberate Operative Rotation of the Maxillo-Mandibular Complex to Alter the A-point to B-point Relationship for Enhanced Facial Esthetics.” J Oral Maxillofac Surg 64: 1687-95.

[16] Parente, E., Lacerda, G., and Silvares, M. G. 2014. "Surgical Manipulation of the Occlusal Plane in Class III Deformities: 5 Features to Help Planning.” Open Journal of Stomatology 4: 238-42. 